\title{
New proofs of some theorems on infinitely differentiable functions: Corrigenda
}

\section{Michael A.B. Deakin}

In a recent note [1], Arkeryd presents a counterexample to my conjecture [2]. The same example appears ir a preprint by von Grudzinski [3], who also points out that the corollary to Theorem 2 does not follow from the proof of the theorem as stated. The fact that $x^{3}+x y^{3}$ and $x^{3}+x y^{3}+y^{3}$ are right equivalent, but not under quasi-identity, provides a counterexample. The proof on $p$. 170 of [2] that $\xi_{i}^{(k+1)}=O(|x|)$ is also incorrect. In order to establish this result, we note that, from equation (31), it suffices to show that $\xi(0, t)=0$ for all $t$. But, from equation $(30), f(\xi(0, t))=0$. If now 0 is an isolated zero of $f$, we are done. If not, we may assume $\xi(0, t)=0$ without loss of generality. The corollary to Theorem 4 shows that this latter case is, in fact, vacuous.

\section{References}

[1] Leif Arkeryd, "A counter-example to a conjecture by Deakin", Buzl. Austral. Math. Soc. 18 (1978), 293-294.

[2] Michael A.B. Deakin, "New proofs of some theorems on infinitely differentiable functions", Bull. Austral. Math. Soc. 17 (1977), $161-175$.

Received 13 June 1978. 
[3] Olaf von Grudzinski, "A note on right-equivalence of map-germs", submitted.

Department of Mathematics,

Monash University,

Clayton,

victoria. 